\title{
Algebras of differentiable functions on Riemannian manifolds
}

\author{
Isabel Garrido, Jesús A. Jaramillo and Yenny C. Rangel
}

\begin{abstract}
For an infinite-dimensional Riemannian manifold $M$ we denote by $C_{b}^{1}(M)$ the space of all real bounded functions of class $C^{1}$ on $M$ with bounded derivative. In this paper we shall see how the natural structure of normed algebra on $C_{b}^{1}(M)$ characterizes the Riemannian structure of $M$, for the special case of the so-called uniformly bumpable manifolds. For that we need, among other things, to extend the classical Myers-Steenrod theorem on the equivalence between metric and Riemannian isometries, to the setting of infinite-dimensional Riemannian manifolds.
\end{abstract}

\section{Introduction}

There is a large number of results in the literature asserting that the topological, metric or differentiable structure of a given space $X$ can be characterized in terms of a suitable algebraic or topological-algebraic structure on the space $C(X)$ of continuous real functions on $X$, or on a certain subfamily of $C(X)$. We should mention, as fundamental prototypes, the classical Banach-Stone theorem from which the topology of a compact space $X$ is determined by the linear metric structure of $C(X)$ (endowed with the sup-norm), and also the classical results of Gelfand-Kolmogorof and Kaplansky asserting, respectively, that the topology of a compact space $X$ is also determined by the algebra structure or by the lattice structure of $C(X)$. We refer to $[\mathbf{3}]$ and references therein for further information about different extensions, generalizations and variants of these results. In this paper, we are interested in the theorem of Myers-Nakai asserting that the Riemannian structure of a (finite-dimensional) manifold $M$ is determined by the natural Banach algebra structure on the the space $C_{b}^{1}(M)$ of all bounded $C^{1}$ functions on $M$ with bounded derivative. This was proved by Myers [9] in the case that $M$ is compact, and later on by Nakai $[\mathbf{1 1}]$ in the general case. Our aim is to give an extension of this result to the setting of infinite-dimensional Riemannian manifolds. The proofs given by Myers and Nakai rely in a strong way on the local compactness of the manifold, and so we have to use different techniques in the infinite-dimensional case. Our approach is to concentrate on the purely metric structure of the manifold. On the one hand, we extend to the infinite-dimensional setting the theorem of Myers and Steenrod [10] about the equivalence between metric and Riemannian isometries on a manifold. On the other hand, we use the techniques developed in [4], for the case of Lipschitz functions, in order to obtain that the Banach algebra structure of $C_{b}^{1}(M)$ determines isometrically the geodesic distance of $M$ for a large class of infinite-dimensional Riemannian manifolds $M$, which includes all separable, connected and complete manifolds. Combining both results we obtain our desired extension of the Myers-Nakai theorem.

\section{The Banach algebra $C_{b}^{1}(M)$}

Throughout $(M, g)$ denotes an infinite-dimensional Riemannian manifold, that is, a $C^{\infty}$ manifold $M$ modeled on some real infinite-dimensional Hilbert space $H$ (see, for example,

Received 5 August 2008; revised 27 May 2009; published online 23 September 2009.

2000 Mathematics Subject Classification 58B20, 46E25, 54C35.

Research supported in part by D.G.I. (Spain) Grant MTM2006-03531. 
[7] for details). For each point $x \in M$, we have that $T_{x} M$ denotes the tangent space of $M$ at $x$, and $0_{x}$ the corresponding null vector in $T_{x} M$. If $f: M \rightarrow \mathbb{R}$ is a real-valued $C^{1}$ function on $M$, then we identify, in the usual way, the differential $d f(x)$ with the gradient $\nabla f(x)$ by means of the scalar product $\langle\cdot, \cdot\rangle_{x}=g_{x}(\cdot, \cdot)$ on $T_{x} M$. That is, for every $v \in T_{x} M$, we have

$$
d f(x)(v)=\langle\nabla f(x), v\rangle_{x}=g_{x}(\nabla f(x), v) .
$$

Now, let $C_{b}^{1}(M)$ be the algebra of all bounded $C^{1}$ functions $f$ on $M$ that have bounded derivative, that is, $\|\nabla f\|_{\infty}:=\sup _{x \in M}\|\nabla f(x)\|_{x}<\infty$. In this way, it is not difficult to check (see $[\mathbf{1}])$ that $C_{b}^{1}(M)$ is a Banach space endowed with the norm as follows:

$$
\|f\|_{C_{b}^{1}}=\sup \left\{\|f\|_{\infty},\|\nabla f\|_{\infty}\right\} .
$$

Moreover, since $\|f \cdot g\|_{C_{b}^{1}} \leqslant 2\|f\|_{C_{b}^{1}} \cdot\|g\|_{C_{b}^{1}}$, it implies that $C_{b}^{1}(M)$ is in fact a Banach algebra with the norm $2\|\cdot\|_{C_{b}^{1}}$.

Note, that if $M$ is connected, then it is, in particular, piecewise $C^{1}$ path connected. In this way for each $x, y \in M$ we can define the geodesic distance dist $(x, y)$, as the infimum of the lengths of all piecewise $C^{1}$ paths in $M$ from $x$ to $y$, where the length of a piecewise $C^{1}$ path $\gamma:[a, b] \rightarrow M$ is given by

$$
\ell(\gamma)=\int_{a}^{b}\left\|\gamma^{\prime}(t)\right\|_{\gamma(t)} d t
$$

Moreover, it is well known that this geodesic distance induces the original topology on $M$, and hence $M$ is, in particular, a metrizable topological space (see, for example, [7]). Throughout we shall always assume that all manifolds are connected. Then we say that $M$ is a complete manifold when $M$, endowed with the geodesic distance, is a complete metric space.

On the other hand, since every $f \in C_{b}^{1}(M)$ has bounded derivative, from the mean-value theorem it follows that $f$ is a Lipschitz function with respect to the geodesic distance. Conversely, if $f \in C^{1}(M)$ is Lipschitz, then $f$ belongs to $C_{b}^{1}(M)$. In fact (see, for example, [1]), we have that $\|\nabla f\|_{\infty}=\operatorname{Lip}(f)$, where $\operatorname{Lip}(f)$ denotes the Lipschitz constant of $f$, that is, we have

$$
\begin{aligned}
\operatorname{Lip}(f) & =\inf \{L \geqslant 0:|f(x)-f(y)| \leqslant L \cdot \operatorname{dist}(x, y) \quad \text { for all } x, y \in M\} \\
& =\sup \left\{\frac{|f(x)-f(y)|}{\operatorname{dist}(x, y)}: \quad x, y \in M, x \neq y\right\} .
\end{aligned}
$$

\section{The Structure Space of $C_{b}^{1}(M)$}

In this section we shall construct the structure space associated to the algebra $C_{b}^{1}(M)$ in an analogous way, as it is done by Isbell in $[6]$ for general algebras of continuous functions, or in [4] for general lattices of continuous functions. First of all, recall that $C_{b}^{1}(M)$ is a unital algebra separating points and closed sets of $M$, and this implies, in particular, that $M$ is endowed with the weak topology given by $C_{b}^{1}(M)$. Moreover, $C_{b}^{1}(M)$ is also closed under bounded inversion, that is, if $f \in C_{b}^{1}(M)$ and $f \geqslant 1$, then $1 / f \in C_{b}^{1}(M)$.

As usual, we say that $\varphi: C_{b}^{1}(M) \rightarrow \mathbb{R}$ is an algebra homomorphism whenever it satisfies the following conditions:

(1) $\varphi(\lambda f+\mu g)=\lambda \varphi(f)+\mu \varphi(g)$

(2) $\varphi(f \cdot g)=\varphi(f) \cdot \varphi(g)$,

for all $f, g \in C_{b}^{1}(M)$ and for all $\lambda, \mu \in \mathbb{R}$. Note that an algebra homomorphism $\varphi$ is nonzero if, and only if, $\varphi(1)=1$. Furthermore, every algebra homomorphism $\varphi$ is positive, that is, $\varphi(f) \geqslant 0$ whenever $f \geqslant 0$. Indeed, when $f$ and $1 / f$ are in $C_{b}^{1}(M)$, then $\varphi(f \cdot(1 / f))=1$ implies that $\varphi(f) \neq 0$ and $\varphi(1 / f)=1 /(\varphi(f))$. Thus, if we assume that $\varphi$ is not positive, there exists 
$f \geqslant 0$ in $C_{b}^{1}(M)$ with $\varphi(f)<0$. The function $g=f-\varphi(f) \geqslant-\varphi(f)>0$ satisfies $g \in C_{b}^{1}(M)$, and also $1 / g \in C_{b}^{1}(M)$ and $\varphi(g)=0$, which is a contradiction.

We define the structure space $H\left(C_{b}^{1}(M)\right)$ as the set of all nonzero algebra homomorphisms $\varphi: C_{b}^{1}(M) \rightarrow \mathbb{R}$, considered as a topological subspace of the product $\mathbb{R}^{C_{b}^{1}(M)}$. It is not difficult to see that $H\left(C_{b}^{1}(M)\right)$ is closed in $\mathbb{R}^{C_{b}^{1}(M)}$. Moreover, since every function in $C_{b}^{1}(M)$ is bounded, it follows that $H\left(C_{b}^{1}(M)\right)$ is in particular a compact space.

Now, consider the natural map $\delta: M \rightarrow H\left(C_{b}^{1}(M)\right)$ given by $\delta(x)=\delta_{x}$, where $\delta_{x}$ is the point evaluation homomorphism, that is, $\delta_{x}(f)=f(x)$ for every $f \in C_{b}^{1}(M)$. Clearly, $\delta$ is a continuous map. On the other hand, the subspace $\delta(M)$ is dense in $H\left(C_{b}^{1}(M)\right)$. Indeed, given $\varphi \in H\left(C_{b}^{1}(M)\right), f_{1}, \ldots, f_{n} \in C_{b}^{1}(M)$, and $\epsilon>0$, there exists some $x \in M$ such that $\mid \delta_{x}\left(f_{i}\right)-$ $\varphi\left(f_{i}\right) \mid<\epsilon$ for all $i=1, \ldots, n$. Otherwise, the function $g=\sum_{i=1}^{n}\left(f_{i}-\varphi\left(f_{i}\right)\right)^{2} \in C_{b}^{1}(M)$ would satisfy $g \geqslant \epsilon$ and $\varphi(g)=0$, and this is impossible since $\varphi$ is positive.

Finally, from the fact that $C_{b}^{1}(M)$ separates points and closed sets of $M$, we can derive that $\delta$ is a topological embedding, and therefore $H\left(C_{b}^{1}(M)\right)$ can be considered as a compactification of $M$. In addition, this compactification has the property that each $f \in C_{b}^{1}(M)$ admits a continuous extension $\hat{f}$ to $H\left(C_{b}^{1}(M)\right)$, namely by defining $\hat{f}(\varphi)=\varphi(f)$ for all $\varphi \in H\left(C_{b}^{1}(M)\right)$. Note that this extension $\hat{f}$ coincides on $H\left(C_{b}^{1}(M)\right)$ with the corresponding projection map $\pi_{f}: \mathbb{R}^{C_{b}^{1}(M)} \rightarrow \mathbb{R}$

Next we see that, for a special class of Riemannian manifolds $M$, those which are complete and uniformly bumpable, the points in $M$ can be topologically distinguished into $H\left(C_{b}^{1}(M)\right)$. The notion of uniformly bumpable manifolds was introduced by Azagra, Ferrera and LópezMesas [1], and it is defined as follows.

Definition 1. A Riemannian manifold $M$ is said to be uniformly bumpable provided there exist numbers $R>1$ and $r>0$ such that for every $x \in M$ and every $\delta \in(0, r)$ there exists a $C^{1}$ (bump) function $b: M \rightarrow[0,1]$ such that the following conditions hold:

(1) $b(x)=1$;

(2) $b(y)=0$ if $\operatorname{dist}(x, y) \geqslant \delta$;

(3) $\|\nabla b\|_{\infty} \leqslant \frac{R}{\delta}$.

We note that this is not a restrictive definition. In fact, as it is proved in [2], every separable Riemannian manifold is uniformly bumpable. On the other hand, we know of no example of a manifold failing to be uniformly bumpable.

Proposition 2. Let $M$ be a complete and uniformly bumpable Riemannian manifold. Then $\varphi \in H\left(C_{b}^{1}(M)\right)$ has a countable neighborhood basis in $H\left(C_{b}^{1}(M)\right)$ if, and only if, $\varphi \in M$.

Proof. First assume that $\varphi \in H\left(C_{b}^{1}(M)\right) \backslash M$ has a countable neighborhood basis. Since $M$ is dense in $H\left(C_{b}^{1}(M)\right)$, it implies that there exists a sequence $\left(x_{n}\right)$ in $M$ converging to $\varphi$. From the completeness of $M$ it follows that $\left(x_{n}\right)$ has no dist-Cauchy subsequence, and therefore there exist $\epsilon>0$ and a subsequence $\left(x_{n_{k}}\right)$ of $\left(x_{n}\right)$ such that $\operatorname{dist}\left(x_{n_{k}}, x_{n_{j}}\right) \geqslant \epsilon$ for $k \neq j$. On the other hand, since $M$ is uniformly bumpable, there exists $R>1$ such that, for some $0<\delta<\epsilon / 2$, we can construct a sequence $\left(b_{k}\right)_{k}$ of $C^{1}$ bump functions satisfying, for each $k \in \mathbb{N}$, the following conditions:

(1) $b_{k}\left(x_{n_{2 k}}\right)=1$;

(2) $b_{k}(y)=0$ if $\operatorname{dist}\left(y, x_{n_{2 k}}\right) \geqslant \delta$;

(3) $\left\|\nabla b_{k}\right\|_{\infty} \leqslant R / \delta$. 
Now, taking $f=\sum_{k} b_{k}$ we have that $f \in C_{b}^{1}(M)$, that $f\left(x_{n_{2 k}}\right)=1$ and that $f\left(x_{n_{2 k+1}}\right)=0$, for every $k$. Therefore, the extended function $\hat{f}$ defined on the whole space $H\left(C_{b}^{1}(M)\right)$ takes the value 1 on $\operatorname{cl}_{H\left(C_{b}^{1}(M)\right)} A$ and the value 0 on $\operatorname{cl}_{H\left(C_{b}^{1}(M)\right)} B$, where $A=\left\{x_{n_{2 k}}: k \in \mathbb{N}\right\}$ and $B=\left\{x_{n_{2 k+1}}: k \in \mathbb{N}\right\}$, and $\mathrm{cl}_{H\left(C_{b}^{1}(M)\right)}$ denotes the closure into $H\left(C_{b}^{1}(M)\right)$. However, this is a contradiction since $\varphi \in \mathrm{cl}_{H\left(C_{b}^{1}(M)\right)} A \cap \mathrm{cl}_{H\left(C_{b}^{1}(M)\right)} B$.

Conversely, if $\varphi \in M$, consider $B_{n}$ the open ball in $M$ with centre $\varphi$ and radius $1 / n$. Then the family $\left\{\mathrm{cl}_{H\left(C_{b}^{1}(M)\right)} B_{n}\right\}$ is easily seen to be a countable neighborhood basis, as required.

\section{Isometries between Riemannian manifolds}

According to a classical result due to Myers and Steenrod [10], the metric and the Riemannian structures on any (finite-dimensional) Riemannian manifold are intimately related, that is, metric isometries and Riemannian isometries coincide. In this section we extend this result to the setting of infinite-dimensional manifolds.

Definition 3. A map $h: M \rightarrow N$ between two Riemannian manifolds $M$ and $N$ is said to be a Riemannian isometry if it is a $C^{1}$ diffeomorphism satisfying

$$
\langle d h(x)(v), d h(x)(w)\rangle_{h(x)}=\langle v, w\rangle_{x}
$$

for every $x \in M$ and every $v, w \in T_{x} M$.

In particular, when $h: M \rightarrow N$ is a Riemannian isometry, then, for every $x \in M$, its differential mapping $d h(x): T_{x} M \rightarrow T_{h(x)} N$ is a linear isometry. Indeed, for every $v \in T_{x} M$, we have that

$$
\|d h(x)(v)\|_{h(x)}^{2}=\langle d h(x)(v), d h(x)(v)\rangle_{h(x)}=\langle v, v\rangle_{x}=\|v\|_{x}^{2} .
$$

From the above, it is clear that every Riemannian isometry preserves the length of piecewise $C^{1}$ paths, and then it is a metric isometry with respect to the respective geodesic distances. Next, we are going to see that the converse is also true.

First we collect some basic facts about geodesics and the exponential map, which can be seen in the book of Lang [7], and which are going to be useful in what follows. Recall that an open subset $U$ of a Riemannian manifold $M$ is said to be convex if, for every $x, y \in U$, there exists a unique (up to reparametrization) geodesic $\gamma$ in $U$ from $x$ to $y$, and such that $\ell(\gamma)=$ $\operatorname{dist}(x, y)$. Note that if, in addition, the geodesic $\gamma:[a, b] \rightarrow M$ is arc-length parametrized, then $\operatorname{dist}\left(\gamma(t), \gamma\left(t^{\prime}\right)\right)=\ell\left(\gamma_{\mid\left[t, t^{\prime}\right]}\right)=\left|t-t^{\prime}\right|$ for every $t, t^{\prime} \in[a, b]$. It is well known that every Riemannian manifold is locally convex, that is, every point in $M$ has a neighborhood basis formed by convex subsets. In fact, a result due to Whitehead asserts that, for every $x \in M$, there exists $r>0$ such that if $0<\delta \leqslant r$, then the open ball $B(x, \delta)$ in $M$ with centre $x$ and radius $\delta$ is a convex subset, and $B(x, \delta)=\exp _{x}\left(B\left(0_{x}, \delta\right)\right)$, where $\exp _{x}$ denotes as usual the exponential map at the point $x$ and $B\left(0_{x}, \delta\right)$ is the corresponding open ball in $T_{x} M$. Moreover, these balls $B(x, \delta)$ in $M$ have the following additional property: for every $y, z \in B(x, \delta)$ the geodesic path from $y$ to $z$ is the unique piecewise $C^{1}$ path joining these points whose length is $\operatorname{dist}(y, z)$.

On the other hand, it is interesting to recall here that the exponential map $\exp _{x}$ satisfies the following: for every $\epsilon>0$ there is $r>0$ such that if $0<\delta<r$, then

$$
\exp _{x}: B\left(0_{x}, \delta\right) \rightarrow B(x, \delta)
$$

is a diffeomorphism and it is $(1+\epsilon)$ bi-Lipschitz (that is, $\exp _{x}$ and $\exp _{x}^{-1}$ are $(1+\epsilon)$-Lipschitz). Moreover, recall that $\exp _{x}$ is a radial isometry, that is, $\|z\|_{x}=\operatorname{dist}\left(x, \exp _{x}(z)\right)$ whenever $z \in$ $B\left(0_{x}, \delta\right)$. 
Using the above remarks we are going to establish the following lemmas.

Lemma 4. Let $M$ be a Riemannian manifold and let $\Gamma:[a, b] \rightarrow M$ be a continuous path such that

$$
\operatorname{dist}\left(\Gamma(t), \Gamma\left(t^{\prime}\right)\right)=\left|t-t^{\prime}\right|
$$

for all $t, t^{\prime} \in[a, b]$. Then $\Gamma$ is a geodesic.

Proof. For each $c \in[a, b]$, let $x=\Gamma(c)$ and let $U_{x}=B(x, r)$ be the convex ball given by the above-mentioned Whitehead's result. We take $\epsilon>0$ such that $\Gamma([c-\epsilon, c+\epsilon] \cap[a, b]) \subset U_{x}$. If $[\alpha, \beta]=[c-\epsilon, c+\epsilon] \cap[a, b], x_{1}=\Gamma(\alpha)$ and $x_{2}=\Gamma(\beta)$, then let $\gamma:\left[0, \operatorname{dist}\left(x_{1}, x_{2}\right)\right] \rightarrow M$ be the unique (arc-length parametrized) geodesic in $U_{x}$ from $x_{1}$ to $x_{2}$. Since $\operatorname{dist}\left(x_{1}, x_{2}\right)=\beta-\alpha$, by a simple translation, we can suppose that $\gamma$ is also an isometry defined on the interval $[\alpha, \beta]$.

Now, for every $y \in \Gamma([\alpha, \beta])$, let $\gamma_{1}$ and $\gamma_{2}$ be the corresponding geodesic curves contained in $U_{x}$ joining $x_{1}$ with $y$, and $y$ with $x_{2}$, respectively. Then, it is clear that the union of $\gamma_{1}$ and $\gamma_{2}$ defines a piecewise $C^{1}$ path whose length is the distance between $x_{1}$ and $x_{2}$, and therefore must coincide with $\gamma$. Then the point $y$ belongs to the set $\gamma([\alpha, \beta])$. In conclusion, we have proved that the set $\Gamma([\alpha, \beta])$ is contained into $\gamma([\alpha, \beta])$ and therefore we can consider the function $\theta=\gamma^{-1} \circ \Gamma:[\alpha, \beta] \rightarrow[\alpha, \beta]$. Since $\theta$ is an isometry such that $\theta(\alpha)=\alpha$ and $\theta(\beta)=\beta$, it must be the identity, and hence $\gamma=\Gamma$ on $[\alpha, \beta]$.

Then $\Gamma$ is locally a geodesic path and therefore it is a geodesic, as desired.

REMARK 5. If in the above lemma we suppose that $\operatorname{dist}\left(\Gamma(t), \Gamma\left(t^{\prime}\right)\right)=C \cdot\left|t-t^{\prime}\right|$, for some $C>0$, then $\Gamma$ is a geodesic parametrized proportionally to arc length.

Lemma 6. Let $M$ be a Riemannian manifold and $x \in M$. Then, we have

$$
\lim _{(v, w) \rightarrow\left(0_{x}, 0_{x}\right)} \frac{\operatorname{dist}\left(\exp _{x} v, \exp _{x} w\right)}{\|v-w\|_{x}}=1 .
$$

Proof. The proof follows at once since, for every $\epsilon>0$, there exists $\delta>0$ such that the map $\exp _{x}: B\left(0_{x}, \delta\right) \rightarrow B(x, \delta)$ is a diffeomorphism $(1+\epsilon)$ bi-Lipschitz.

Next we give the announced Myers-Steenrod result. For the proof, we follow the lines of Helgason [5] for the finite-dimensional case.

Theorem 7. Let $h: M \rightarrow N$ be a bijection between two Riemannian manifolds $M$ and $N$ that preserves the corresponding geodesic distances. Then $h$ is a Riemannian isometry.

Proof. Let $x \in M$ and $y=h(x) \in N$. Consider $r>0$ such that $B(x, r)$ and $B(y, r)$ are convex neighborhoods of $x$ and $y$, respectively, and such that the corresponding exponential maps $\exp _{x}: B\left(0_{x}, r\right) \rightarrow B(x, r)$ and $\exp _{y}: B\left(0_{y}, r\right) \rightarrow B(y, r)$ are diffeomorphisms of class $C^{1}$. Since $h$ preserves the geodesic distances, it follows that $h$ is a bijection from $B(x, r)$ onto $B(y, r)$.

Now, for every $v \in T_{x} M$ with $v \neq 0$, consider the geodesic in $B(x, r)$ with speed $\|v\|_{x}$, that is, $\gamma(t)=\exp _{x}(t v)$, defined for $-r /\|v\|_{x}<t<r /\|v\|_{x}$. Then, the continuous path $\Gamma(t)=h(\gamma(t))$ is contained in $B(y, r)$ and satisfies

$$
\operatorname{dist}\left(\Gamma(t), \Gamma\left(t^{\prime}\right)\right)=\operatorname{dist}\left(\gamma(t), \gamma\left(t^{\prime}\right)\right)=\|v\|_{x} \cdot\left|t-t^{\prime}\right|
$$


when $t$ and $t^{\prime}$ belong to the interval $\left(-r /\|v\|_{x}, r /\|v\|_{x}\right)$. Now, by Remark 5 , it is clear that $\Gamma$ is a geodesic parametrized proportionally to arc length. In particular, $\Gamma$ is differentiable.

For $v \in T_{x} M$, with $v \neq 0$, let $v^{\prime} \in T_{y} N$ be the tangent vector to $\Gamma$ at the point $y$. Note that $\Gamma$ is the geodesic in $y$ with the tangent vector $v^{\prime}$, and then $\Gamma(t)=\exp _{y}\left(t v^{\prime}\right)$ when $t \in$ $\left(-r /\left\|v^{\prime}\right\|_{y}, r /\left\|v^{\prime}\right\|_{y}\right)$. Since $h$ preserves distances, it follows that both geodesics $\gamma$ and $\Gamma$ have the same speed, that is, $\|v\|_{x}=\left\|v^{\prime}\right\|_{y}$.

In this way, if we put $h^{\prime}\left(0_{x}\right)=0_{y}$, and $h^{\prime}(v)=v^{\prime}$, then we have defined a function $h^{\prime}: T_{x} M \rightarrow$ $T_{y} N$, such that $\left\|h^{\prime}(v)\right\|_{y}=\|v\|_{x}$. Moreover, it is not difficult to check that $h^{\prime}$ is also positively homogeneous, that is, $h^{\prime}(\lambda v)=\lambda h^{\prime}(v)$ for $\lambda>0$.

In order to see that $h^{\prime}$ is linear, we shall use the classical Mazur-Ulam theorem [8]. For that it is enough to see that $h^{\prime}$ is an isometry between Banach spaces, that is, $\left\|h^{\prime}(v)-h^{\prime}(w)\right\|_{y}=\| v-$ $w \|_{x}$ for every $v, w \in T_{x}(M)$. Indeed, let $v, w \in T_{x}(M)$ and let $\lambda>0$ be such that $\|\lambda v\|_{x}<r$ and $\|\lambda w\|_{x}<r$; then using Lemma 6 , we have

$$
\begin{aligned}
\frac{2\langle v, w\rangle_{x}}{\|v\|_{x}\|w\|_{x}} & =\frac{\|v\|_{x}^{2}+\|w\|_{x}^{2}}{\|v\|_{x}\|w\|_{x}}-\frac{\|\lambda v-\lambda w\|_{x}^{2}}{\|\lambda v\|_{x}\|\lambda w\|_{x}} \\
& =\frac{\|v\|_{x}^{2}+\|w\|_{x}^{2}}{\|v\|_{x}\|w\|_{x}}-\lim _{\lambda \rightarrow 0^{+}} \frac{\operatorname{dist}\left(\exp _{x} \lambda v, \exp _{x} \lambda w\right)^{2}}{\|\lambda v\|_{x}\|\lambda w\|_{x}} .
\end{aligned}
$$

Since $h^{\prime}$ preserves the right-hand side, for all $v, w \in T_{x} M$, it follows that

$$
\left\langle h^{\prime}(v), h^{\prime}(w)\right\rangle_{y}=\langle v, w\rangle_{x}
$$

Then $h^{\prime}$ preserves the scalar product, and therefore it is a linear isometry. Finally, taking into account that on $B(x, r)$ we have $h=\exp _{y} \circ h^{\prime} \circ \exp _{x}^{-1}$, and that $h^{\prime}$ is a diffeomorphism between $T_{x} M$ and $T_{y} N$, we derive that $h$ is differentiable at the point $x$, and in fact $d h(x)=h^{\prime}$. We finish doing the same for $h^{-1}$.

\section{A Myers-Nakai theorem}

In this section we see how, for a large class of infinite-dimensional Riemannian manifolds $M$, the structure of normed algebra of $C_{b}^{1}(M)$ determines the Riemannian structure of $M$. In this way we will prove a Banach-Stone type theorem in the context of infinite-dimensional Riemannian manifolds, which provides an infinite-dimensional version of the corresponding result by Myers [9] and Nakai [11].

First we are going to give a simple regularization lemma on Hilbert spaces.

Lemma 8. Let $H$ be a Hilbert space and let $r>0$. For every $\epsilon>0$, there exists a function $\psi: H \rightarrow \mathbb{R}$ such that the following conditions hold:

(1) $\psi(0)=r$;

(2) $\operatorname{supp}(\psi) \subset B(0, r)$;

(3) $\|\psi\|_{\infty} \leqslant r$;

(4) $\operatorname{Lip}(\psi) \leqslant 1+\epsilon$;

(5) $\psi \in C_{b}^{1}(H)$;

(6) $\|x\| \leqslant r-\psi(x)+\epsilon$, when $x \in B(0, r)$.

Proof. Given $\epsilon>0$, consider a $C^{\infty}$ function $\theta: \mathbb{R} \rightarrow[0, r]$ such that the following properties hold:

(i) $\theta=0$ on a neighborhood of the interval $(-\infty, 0]$;

(ii) $\theta=r$ on a neighborhood of the interval $[r,+\infty)$; 
(iii) $|t-\theta(t)| \leqslant \epsilon$, for every $t \in[0, r]$;

(iv) $\left|\theta^{\prime}(t)\right| \leqslant 1+\epsilon$, for every $t \in \mathbb{R}$.

Then it is enough to define $\psi(x)=r-\theta(\|x\|)$, for $x \in H$.

From our next result we deduce that if a composition operator from $C_{b}^{1}(N)$ to $C_{b}^{1}(M)$ is a linear isometry, then it is induced by a metric isometry from $M$ to $N$.

Theorem 9. Let $M$ and $N$ be Riemannian manifolds and let $h: M \rightarrow N$ be a map such that the composition operator $T: C_{b}^{1}(N) \rightarrow C_{b}^{1}(M)$, given by $T(f)=f \circ h$, is continuous. Then $h$ is $\|T\|$-Lipschitz with respect to the corresponding geodesic distances.

Proof. First note that $h$ must be continuous since $M$ and $N$ are endowed with the weak topology given by $C_{b}^{1}(M)$ and $C_{b}^{1}(N)$, respectively (see Section 3). Now, in order to see that $h$ is in fact Lipschitz, let $x_{1}, x_{2} \in M$ and $\epsilon>0$. Consider $\sigma:[0,1] \rightarrow M$ a piecewise $C^{1}$ path in $M$ from $x_{1}$ to $x_{2}$, with $\ell(\sigma) \leqslant \operatorname{dist}\left(x_{1}, x_{2}\right)+\epsilon$. Since $h$ is continuous, it follows that $\hat{\sigma}=$ $h \circ \sigma:[0,1] \rightarrow N$ is a continuous path in $N$ from $h\left(x_{1}\right)$ to $h\left(x_{2}\right)$. Thus, for every $y \in \hat{\sigma}([0,1])$, let $0<r_{y}<1$ such that $\exp _{y}: B\left(0_{y}, r_{y}\right) \rightarrow B\left(y, r_{y}\right)$ is a diffeomorphism $(1+\epsilon)$ bi-Lipschitz on the corresponding convex open balls.

Next, for each $t \in[0,1]$, consider an open (in $[0,1]$ ) interval $I_{t}$ around $t$ and such that $\hat{\sigma}\left(I_{t}\right) \subset B\left(\hat{\sigma}(t), r_{\hat{\sigma}(t)}\right)$. Using a connectedness argument we can extract from the open covering $\left\{I_{t}\right\}_{t \in[0,1]}$ a simple chain connecting 0 and 1 , that is, there exists a finite sequence $I_{t_{1}}, \ldots$, $I_{t_{m}}$ such that $0=t_{1}<\ldots<t_{m}=1$, and $I_{t_{j}} \cap I_{t_{k}} \neq \emptyset$ if, and only if, $|j-k| \leqslant 1$. Note that $I_{t_{1}} \cup \ldots \cup I_{t_{m}}=[0,1]$. It is easy to see that, for each $i=1, \ldots,(m-1)$, we can choose a point $s_{i} \in I_{t_{i}} \cap I_{t_{i+1}}$, with $t_{i}<s_{i}<t_{i+1}$.

Now we apply Lemma 8 to each $r_{y}$ with the same $\epsilon / 2 m$, and we get the corresponding functions $\psi_{y} \in C_{b}^{1}(H)$. If we define the function $f_{y}=\psi_{y} \circ \exp _{y}^{-1}$ on $B\left(y, r_{y}\right)$ and $f_{y}=0$ on $N \backslash B\left(y, r_{y}\right)$, then we have that the following conditions hold:

(1) $f_{y}(y)=r_{y}$;

(2) $\left\|f_{y}\right\|_{\infty} \leqslant r_{y} \leqslant 1$

(3) $\operatorname{Lip}\left(f_{y}\right) \leqslant(1+\epsilon)^{2}$;

(4) $f_{y} \in C_{b}^{1}(N)$;

(5) $\operatorname{dist}(y, z) \leqslant f_{y}(y)-f_{y}(z)+(\epsilon / 2 m)$, whenever $z \in B\left(y, r_{y}\right)$.

Moreover, from the continuity of the operator $T$, it follows that

$$
\operatorname{Lip}\left(f_{y} \circ h\right) \leqslant\left\|f_{y} \circ h\right\|_{C_{b}^{1}(M)}=\left\|T\left(f_{y}\right)\right\|_{C_{b}^{1}(M)} \leqslant\|T\| \cdot\left\|f_{y}\right\|_{C_{b}^{1}(N)} \leqslant\|T\| \cdot(1+\epsilon)^{2} .
$$

Finally, with all the above ingredients, if we denote $y_{i}=h\left(\sigma\left(t_{i}\right)\right)$ for $i=1, \ldots, m$, then we obtain that

$$
\begin{aligned}
\operatorname{dist}\left(h\left(x_{1}\right), h\left(x_{2}\right)\right) \leqslant & \sum_{i=1}^{m-1}\left\{\operatorname{dist}\left(h\left(\sigma\left(t_{i}\right)\right), h\left(\sigma\left(s_{i}\right)\right)\right)+\operatorname{dist}\left(h\left(\sigma\left(s_{i}\right)\right), h\left(\sigma\left(t_{i+1}\right)\right)\right)\right\} \\
\leqslant & \sum_{i=1}^{m-1}\left\{f_{y_{i}}\left(y_{i}\right)-f_{y_{i}}\left(h\left(\sigma\left(s_{i}\right)\right)\right)+f_{y_{i+1}}\left(y_{i+1}\right)-f_{y_{i+1}}\left(h\left(\sigma\left(s_{i}\right)\right)\right)+\frac{\epsilon}{m}\right\} \\
\leqslant & \sum_{i=1}^{m-1}\left\{\operatorname{Lip}\left(f_{y_{i}} \circ h\right) \operatorname{dist}\left(\sigma\left(t_{i}\right), \sigma\left(s_{i}\right)\right)\right. \\
& \left.\quad+\operatorname{Lip}\left(f_{y_{i+1}} \circ h\right) \operatorname{dist}\left(\sigma\left(t_{i+1}\right), \sigma\left(s_{i}\right)\right)+\frac{\epsilon}{m}\right\}
\end{aligned}
$$




$$
\begin{aligned}
& \leqslant \sum_{i=1}^{m-1}\|T\|(1+\epsilon)^{2}\left\{\operatorname{dist}\left(\sigma\left(t_{i}\right), \sigma\left(s_{i}\right)\right)+\operatorname{dist}\left(\sigma\left(s_{i}\right), \sigma\left(t_{i+1}\right)\right)\right\}+\epsilon \\
& \leqslant \sum_{i=1}^{m-1}\|T\|(1+\epsilon)^{2} \cdot \ell\left(\left.\sigma\right|_{\left[t_{i}, t_{i+1}\right]}\right)+\epsilon \\
& =\|T\|(1+\epsilon)^{2} \cdot \ell(\sigma)+\epsilon \leqslant\|T\|(1+\epsilon)^{2} \cdot\left(\operatorname{dist}\left(x_{1}, x_{2}\right)+\epsilon\right)+\epsilon,
\end{aligned}
$$

and, therefore $h$ is $\|T\|$-Lipschitz.

We finish with the announced Myers-Nakai theorem in the infinite-dimensional setting. Recall that two normed algebras $\left(A,\|\cdot\|_{A}\right)$ and $\left(B,\|\cdot\|_{B}\right)$ are said to be equivalent as normed algebras whenever there exists an algebra isomorphism $T: A \rightarrow B$ that is a norm-isometry, that is, $\|T(a)\|_{B}=\|a\|_{A}$, for every $a \in A$.

Theorem 10. Let $M$ and $N$ be complete Riemannian manifolds that are uniformly bumpable. Then $M$ and $N$ are equivalent as Riemannian manifolds if, and only if, $C_{b}^{1}(M)$ and $C_{b}^{1}(N)$ are equivalent as normed algebras. Moreover, every normed algebra isomorphism $T: C_{b}^{1}(N) \rightarrow C_{b}^{1}(M)$ is of the form $T(f)=f \circ h$, where $h: M \rightarrow N$ is a Riemannian isometry.

Proof. First, if $h: M \rightarrow N$ is a Riemannian isometry, then it is easy to check that the composition operator $T: C_{b}^{1}(N) \rightarrow C_{b}^{1}(M)$, defined by $T(f)=f \circ h$, gives the equivalence between the corresponding normed algebras $C_{b}^{1}(M)$ and $C_{b}^{1}(N)$.

Conversely, suppose $T: C_{b}^{1}(N) \rightarrow C_{b}^{1}(M)$ is an isometry between normed algebras. Then, consider the function $h: H\left(C_{b}^{1}(M)\right) \rightarrow H\left(C_{b}^{1}(N)\right)$ between the structure spaces (see Section 3 ) given by $h(\varphi)=\varphi \circ T$ for every $\varphi \in H\left(C_{b}^{1}(M)\right)$. Then $h$ is a bijection and in fact $h$ is a homeomorphism since $\pi_{f} \circ h=\pi_{T(f)}$, for every $f \in C_{b}^{1}(N)$, where $\pi_{f}$ and $\pi_{T(f)}$ denote the projection maps on the corresponding product spaces.

Now, by applying Proposition 2, we obtain that a point $\varphi \in H\left(C_{b}^{1}(M)\right)$ has a countable neighborhood basis in $H\left(C_{b}^{1}(M)\right)$ if, and only if, $\varphi \in M$ and the same holds in the complete manifold $N$. Therefore the homeomorphism $h$ takes $M$ onto $N$.

Moreover, for every $x \in M$ and every $f \in C_{b}^{1}(N)$, we have that

$$
T(f)(x)=\delta_{x}(T(f))=\left(\delta_{x} \circ T\right)(f)=h\left(\delta_{x}\right)(f)=h(f(x))=(h \circ f)(x)
$$

and then it follows that $T(f)=h \circ f$. Now, from Theorem 9 we can deduce that $h: M \rightarrow N$ is $\|T\|$-Lipschitz with respect to the geodesic distances. Since $T$ is an isometry, then $\|T\|=1$, and hence $h$ is 1-Lipschitz. Now making the same with $h^{-1}$, we have that $h^{-1}$ is also 1-Lipschitz, and hence $h$ is a metric isometry. Finally, from Theorem 7, we find that $h$ is in fact a Riemannian equivalence.

\section{References}

1. D. Azagra, J. Ferrera and F. López-Mesas, 'Nonsmooth analysis and Hamilton-Jacobi equations on Riemannian manifolds', J. Funct. Anal. 220 (2005) 304-361.

2. D. Azagra, J. Ferrera, F. López-Mesas and Y. Rangel, 'Smooth approximation of Lipschitz functions on Riemannian manifolds', J. Math. Anal. Appl. 326 (2007) 1370-1378.

3. M. I. GARRido and J. A. JARAmillo, 'Variations on the Banach-Stone theorem', Extracta Math. 17 (2002) $351-383$.

4. M. I. Garrido and J. A. Jaramillo, 'Homomorphisms on function lattices', Monatsh. Math. 141 (2004) $127-146$.

5. S. Helgason, Differential geometry, Lie groups, and symmetryc spaces (Academic Press, New York, 1978).

6. J. R. Isbell, 'Algebras of uniformly continuous functions', Ann. of Math. (2) 69 (1958) 96-125. 
7. S. LANG, Fundaments of differential geometry, Graduate Text in Mathematics 191 (Springer, New York, 1999).

8. S. Mazur and S. Ulam, 'Sur les transformations isométriques d'espaces vectoriels normés', C. R. Math. Acad. Sci. Paris 194 (1932) 946-948.

9. S. B. Myers, 'Algebras of differentiable functions', Proc. Amer. Math. Soc. 5 (1954) 917-922.

10. S. B. Myers and N. E. SteEnrod, 'The group of isometries of a Riemannian manifold', Ann. of Math. (2) 40 (1939) 400-416

11. M. NAKAI, 'Algebras of some differentiable functions on Riemannian manifolds', Japan. J. Math. 29 (1959) 60-67.

\section{Isabel Garrido}

Departamento de Geometría y Topología Universidad Complutense de Madrid 28040 Madrid

Spain

maigarri@mat.ucm.es
Jesús A. Jaramillo and Yenny C. Rangel Departamento de Análisis Matemático Universidad Complutense de Madrid 28040 Madrid

Spain

jaramil@mat.ucm.es yenny.rangel@mat.ucm.es 